\title{
Direct detection of a substellar companion to the young nearby star PZ Telescopii ${ }^{\star}$
}

\author{
M. Mugrauer ${ }^{1}$, N. $\operatorname{Vogt}^{2,3}$, R. Neuhäuser ${ }^{1}$, and T. O. B. Schmidt ${ }^{1}$ \\ 1 Astrophysikalisches Institut und Universitäts-Sternwarte Jena, Schillergäßchen 2, 07745 Jena, Germany \\ e-mail: markus@astro.uni-jena.de \\ 2 Departamento de Física y Astronomía, Universidad de Valparaíso, Avenida Gran Bretaña 1111, Valparaíso, Chile \\ 3 Instituto de Astronomía, Universidad Catolica del Norte, Avda. Angamos 0610, Antofagasta, Chile
}

Received 4 August 2010 / Accepted 25 August 2010

\section{ABSTRACT}

\begin{abstract}
Aims. We study the formation of substellar objects (exoplanets and brown dwarfs) that are companions to young nearby stars. Methods. With high-contrast AO imaging obtained with NACO at ESO VLT, we search for faint companion candidates around our targets, whose companionship can be confirmed with astrometry.

Results. We identified a faint substellar companion to the nearby pre-main sequence star PZTel, a member of the $\beta$ Pic moving group. The companion is 5-6 mag fainter than its host star in $J H K$ and is located at a separation of only $0.3 \operatorname{arcsec}$ (or 15 AU of projected separation) northeast of PZ Tel. Within three NACO observing epochs, we can confirm the common proper motion $(>39 \sigma)$ and detected orbital motion of PZ Tel B around its primary $(>37 \sigma)$. The photometry of the newly found companion is consistent with a brown dwarf with a mass between 24 and $40 M_{\text {Jup }}$, at the distance $(50 \mathrm{pc})$ and age $(8-20 \mathrm{Myr})$ of PZ Tel. The effective temperature of the companion, derived from its photometry, ranges between 2500 and $2700 \mathrm{~K}$, which corresponds to a spectral type between M6 and M8. After $\beta$ Pic b, PZ Tel B is the second closest substellar companion imaged directly around a young star.
\end{abstract}

Key words. stars: individual: PZ telescopii - brown dwarfs

\section{Introduction}

For several years, we have performed a high-contrast imaging search for substellar companions of young nearby stars using the adaptive optics and infrared imager NACO, which is installed at the Nasmyth-platform of UT4 (Yepun) at ESO's Paranal Observatory, in Chile. In our ongoing imaging campaign, we observed PZ Tel (HD 174429, HIP 92680), which is a solar-analog pre-main-sequence star of spectral type K0Vp, at a distance of $49.7 \pm 2.9 \mathrm{pc}$ (Perryman et al. 1997), which has a mass of $1.02 \pm 0.04 M_{\odot}$ (see Allende Prieto \& Lambert 1999). Randich et al. (1993) first reported the rapid rotational velocity $\left(v \sin (i)=70 \mathrm{~km} \mathrm{~s}^{-1}\right)$ and lithium abundance $(N(\mathrm{Li})=3.9)$ of this star, which are both important youth indicators. These results were later confirmed by others (see e.g. Soderblom et al. 1998; Rocha-Pinto et al. 2002; Barnes et al. 2000). Because of its space motion $\left([U, V, W]=[-10.6,-15.4,-7.9] \mathrm{km} \mathrm{s}^{-1}\right)$ and its other youth indicators, Zuckerman et al. (2001) and Torres et al. (2006) proposed that PZ Tel is a member of the $\beta$ Pic moving group, with an age of $12_{-4}^{+8}$ Myr. Tetzlaff et al. (2010) determined the age of PZ Tel by comparing its luminosity and temperature with several theoretical model isochrones yielding an age of $12.8 \pm 2.2 \mathrm{Myr}$, consistent with the general age estimate for the $\beta$ Pic moving group. Rebull et al. (2008) reported the detection of excess emission in the spectral energy distribution of PZ Tel at $70 \mu \mathrm{m}$ with MIPS/Spitzer. These observations indicate that the star is surrounded by a cold $(\sim 40 \mathrm{~K})$ low-mass dust disk with a total mass of only $\sim 0.3 M_{\text {Moon }}$, which extends from its

\footnotetext{
* Based on observations obtained at Paranal Observatory in ESO programs 079.C-0908(A), 083.C-0150(B), 085.C-0012(A).
}

Table 1. Observation log for all imaging data presented.

\begin{tabular}{lclccc}
\hline \hline Optics & Epoch & Filter & $D I T[\mathrm{~s}]$ & $N D I T$ & $N$ \\
\hline S27 & $2007 / 06 / 13$ & $K_{\mathrm{s}}+N D_{\text {Short }}$ & 1.000 & 10 & 5 \\
S13 & $2009 / 09 / 28$ & $K_{\mathrm{s}}$ & 0.347 & 122 & 42 \\
S13 & $2010 / 05 / 05$ & $J$ & 0.347 & 100 & 10 \\
S13 & $2010 / 05 / 05$ & $H+N D_{\text {Short }}$ & 5.000 & 10 & 10 \\
S13 & $2010 / 05 / 05$ & $K_{\mathrm{s}}+N D_{\text {Short }}$ & 5.000 & 10 & 10 \\
S13 & $2010 / 05 / 06$ & $J+N D_{\text {Short }}$ & 5.000 & 10 & 10 \\
S13 & $2010 / 05 / 07$ & $J+N D_{\text {Short }}$ & 5.000 & 10 & 30 \\
S13 & $2010 / 05 / 07$ & $H+N D_{\text {Short }}$ & 5.000 & 10 & 21 \\
S13 & $2010 / 05 / 07$ & $K_{\mathrm{s}}+N D_{\text {Short }}$ & 5.000 & 10 & 9 \\
\hline
\end{tabular}

Notes. With the detector integration time (DIT), the number of detector integrations per image (NDIT), as well as the number of frames $(N)$ taken in total.

inner border at a separation of about $35 \mathrm{AU}$ to $200 \mathrm{AU}$ at its outer edge.

\section{Observations: astrometry and photometry}

We first imaged PZ Tel in September 2009 with NACO's S13 optics in the $K_{\mathrm{s}}$-band. The imaging-setup of all observations is listed in Table 1.

Because of the brightness of PZ Tel $\left(K_{\mathrm{S}} \sim 6.4 \mathrm{mag}\right)$, we consistently used the shortest possible detector integration time

${ }^{1}$ Public data from the ESO data archive, taken in ESO program 079.C0908(A). 


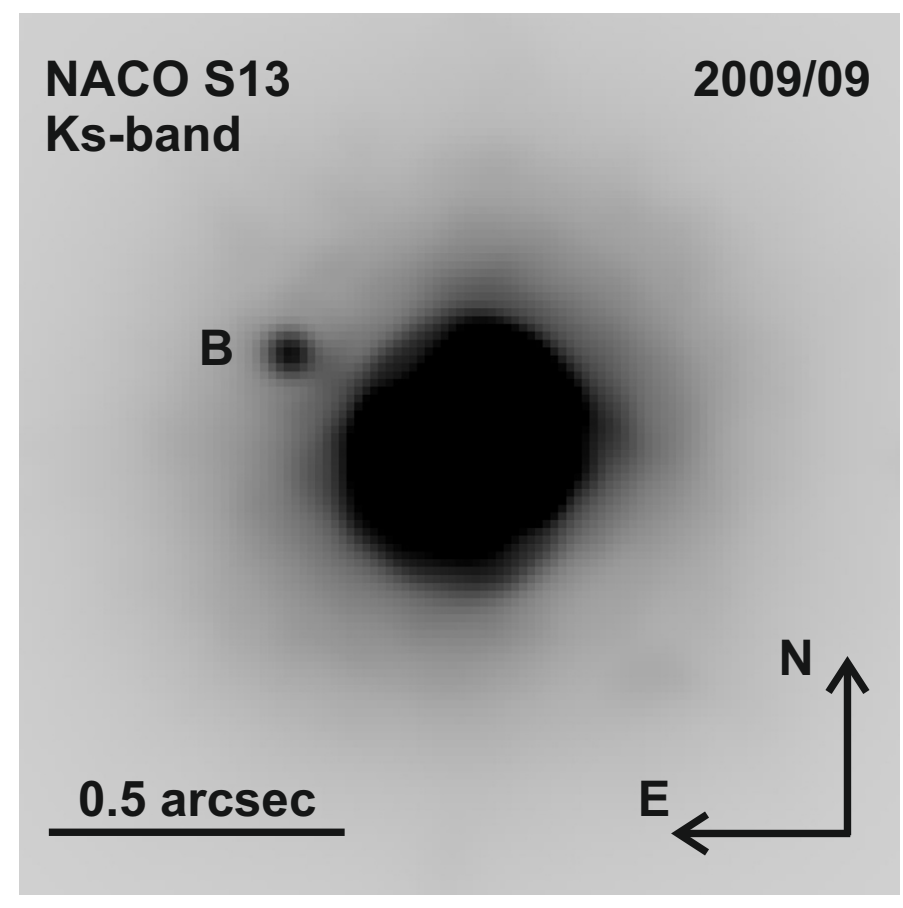

Fig. 1. This pattern shows our deep NACO image of PZ Tel and its faint $\left(\Delta K_{\mathrm{s}}>5 \mathrm{mag}\right)$ companion-candidate, taken in September 2009. The total integration time is $29.6 \mathrm{~min}$ in the $K_{\mathrm{s}}$-band.

$($ DIT $=0.347 \mathrm{~s})$ to limit saturation only to the central few pixels of the point spread function (PSF) of the bright star, and 122 of these integrations were then averaged to produce one image. To subtract the bright sky background in the $K_{\mathrm{s}}$-band, the telescope position was then moved between the individual images, and 42 images were taken in total, within a jitter-box with a width of 7 arcsec. Internal lamp flats and skyflats were used to calibrate the individual pixel sensitivity of NACO's infrared detector. The background estimation and subtraction, as well as the flat-fielding of all images, was then performed with the ESO package ECLIPSE ${ }^{2}$ (Devillard 2001), which finally also combined all images, using a shift+add procedure. The fully reduced NACO image, taken in September 2009, is shown in Fig. 1.

In our deep NACO observation beside the bright central star PZ Tel, we detected a faint $\left(\Delta K_{\mathrm{s}}>5 \mathrm{mag}\right)$ companion-candidate, which is located only $\sim 0.3$ arcsec northeast of the star. To test whether this object is a true companion of the star or only a background source, which is merely randomly located close to but far behind PZ Tel, data for additional observing epochs would be needed, in which the candidate is imaged with PZ Tel. Therefore, we searched first in the ESO data archive for additional NACO observations of PZ Tel, and found a data-set in which the candidate is clearly detected. The public archival data were taken in June 2007 with NACO's S27 optics through the $K_{\mathrm{s}}$-band filter in combination with NACO's neutral density filter $N D_{\text {Short }}$ (transmission of about $1.4 \%$ ).

To determine accurate astrometry of the candidate relative to PZ Tel, images of astrometric calibrators had to be taken in each observing run. For this purpose, in September 2009, we observed the globular cluster 47 Tuc for which precise HST astrometry is available for several of its members. We determined the pixel scale PS and the position angle PA of NACO's S13 optics, which is listed in Table 2 with the astrometric calibration of the archival data, taken from Chauvin et al. (2010).

\footnotetext{
${ }^{2}$ ESO C Library for an Image Processing Software Environment.
}

Table 2. The astrometric calibration of NACO for all observing epochs whose data are presented in this letter.

\begin{tabular}{lccc}
\hline \hline Optics & Epoch & PS [mas/pixel] & PA [ $\left.{ }^{\circ}\right]$ \\
\hline S27 & $2007 / 06$ & $27.01 \pm 0.05$ & $-0.06 \pm 0.15$ \\
S13 & $2009 / 09$ & $13.234 \pm 0.018$ & $+0.42 \pm 0.10$ \\
S13 & $2010 / 05$ & $13.231 \pm 0.020$ & $+0.67 \pm 0.13$ \\
\hline
\end{tabular}

Notes. The pixel scale PS and the detector position angle PA are listed with their uncertainties.

Table 3. The angular separations (sep) and position angles (PA) of the detected close companion-candidate relative to PZTel for all NACO observing epochs.

\begin{tabular}{lcccc}
\hline \hline Optics \& epoch & sep [mas] & sep $_{\text {bg }}[\mathrm{mas}]$ & $s_{\text {bg }}[\sigma]$ & $s_{\text {orb }}[\sigma]$ \\
\hline S27 2007/06/13 & $254.6 \pm 2.5$ & - & - & - \\
S13 2009/09/28 & $336.9 \pm 1.5$ & $373.8 \pm 4.7$ & 8 & 28 \\
S13 2010/05/05 & $356.3 \pm 1.1$ & $394.6 \pm 5.3$ & 7 & 37 \\
S13 2010/05/07 & $354.8 \pm 1.3$ & $395.4 \pm 5.3$ & 7 & 36 \\
\hline Optics \& epoch & PA $\left[^{\circ}\right]$ & PA $_{\text {bg }}\left[^{\circ}\right]$ & $s_{\text {bg }}[\sigma]$ & $S_{\text {orb }}[\sigma]$ \\
S27 2007/06/13 & $61.74 \pm 0.58$ & - & - & - \\
S13 2009/09/28 & $60.55 \pm 0.22$ & $34.77 \pm 0.84$ & 30 & 2 \\
S13 2010/05/05 & $60.47 \pm 0.21$ & $24.89 \pm 0.89$ & 39 & 2 \\
S13 2010/05/07 & $60.41 \pm 0.21$ & $24.88 \pm 0.89$ & 39 & 2 \\
\hline
\end{tabular}

Notes. In the columns sep $\mathrm{pg}_{\mathrm{g}}$ and $\mathrm{PA}_{\mathrm{bg}}$, we show the expected separations and position angles in the case that the detected candidate is a non-moving background object. The significance level on which this background hypothesis can be rejected is listed in column $s_{\mathrm{bg}}$, and the one of detected orbital motion in column $s_{\text {orb }}$.

With the given astrometric calibration, we could then determine the relative astrometry of the companion-candidate, i.e. its angular separation (sep) and position angle (PA) to PZ Tel, in all available observing epochs. Because of the small separation of the candidate from the much brighter star, the PSF of PZ Tel was always removed in all images with spatial filtering, to determine the relative astrometry of the candidate. All astrometric measurements are summarized in Table 3 and illustrated in Fig. 2.

The comparison of our NACO data from September 2009 with the archival data from June 2007 already clearly indicates that the detected companion-candidate is not a non-moving background source. The expected separations and position angles of such an object can be derived (see Table 3, and Fig. 2) from the relative astrometry of the candidate, the given epoch differences, as well as the proper and parallactic motion of PZ Tel, which are well known from Hipparcos measurements ${ }^{4}$. We find that the position angle of the candidate only slightly decreases between both observing epochs by $1.19 \pm 0.62^{\circ}(\mathrm{PA}=$ $-0.5 \pm 0.3^{\circ} / \mathrm{yr}$ ), while a decrease of about $27^{\circ}$ is expected for a non-moving background source. Hence, we can reject the background hypothesis for the detected candidate on the basis of its position angle measurements (at a significance level $>39 \sigma$ ). While the change in the position angle is relatively small, the separation of the candidate increases significantly between both observing epochs by $82.3 \pm 2.9$ mas ( $\mathrm{sep}=35.8 \pm 1.3 \mathrm{mas} / \mathrm{yr}$ ). However, even this high relative motion can be attributed to orbital motion around PZ Tel (see Sect. 4 for a discussion). We

\footnotetext{
3 Astrometric calibration of public archival data from ESO program 079.C-0908(A), listed in Chauvin et al. (2010).

${ }^{4} \mu_{\alpha} \cos (\delta)=16.64 \pm 1.32 \mathrm{mas} / \mathrm{yr}, \mu_{\delta}=-83.58 \pm 0.87 \mathrm{mas} / \mathrm{yr}$, and $\pi=20.14 \pm 1.18$ mas (Perryman et al. 1997).
} 

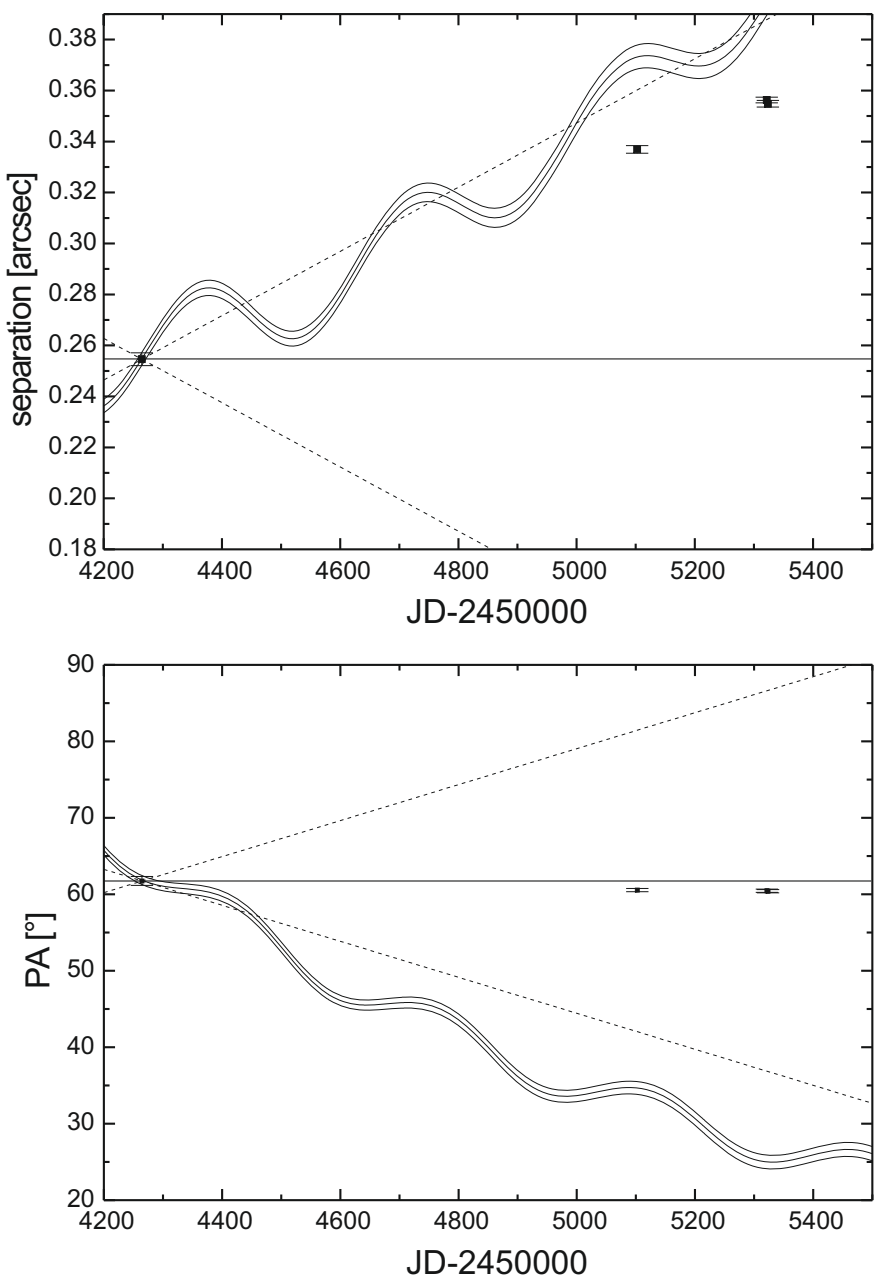

Fig. 2. The angular separation and position angle of the detected candidate for all observing epochs. The wobbled lines illustrate the expected change in both quantities in the case that the candidate is a non-moving background object. The dashed straight lines show the expected maximal change in both parameters for an object on a gravitationally bound orbit around PZ Tel.

therefore conclude that the detected candidate is a real companion of PZ Tel, and denote it hereinafter as PZ Tel B.

To follow the orbital motion of PZ Tel B around its primary, we observed the PZ Tel system in May 2010 again with NACO in the $K_{\mathrm{s}}$-band. For the astrometric calibration, images of the globular cluster 47 Tuc were taken, as well as each night images of the wide binaries HIP 42758, HIP 45072, HIP 46657, and HIP 50602. The instrument is found to be stable within the individual nights in May 2010. In addition to our $K_{\mathrm{s}}$-band observations, we also obtained images in the $J$-, and $H$-band to determine the infrared photometry of PZ TelB. The magnitude difference between PZTelB and its primary was consistently measured after the subtraction of the PSF of the bright star in all NACO images, and is summarized in Table 4.

Since PZTel A is saturated in our deep NACO images, taken in September 2009, only a lower limit to the magnitudedifference in the $K_{\mathrm{s}}$-band could be derived $\left(\Delta K_{\mathrm{s}}>5.0 \pm 0.1 \mathrm{mag}\right)$. The determined limit agrees with the photometry of PZ Tel B obtained in all other observing epochs in which NACO's neutral density filter $N D_{\text {Short }}$ was used if PZ Tel A would have saturated the NACO detector. The photometric measurements from the
Table 4. Photometry of PZ Tel B.

\begin{tabular}{ccll}
\hline \hline Optics & Epoch & Filter & $\Delta m[\mathrm{mag}]$ \\
\hline S13 & $2010 / 05 / 05$ & $J$ & $5.54 \pm 0.20$ \\
S13 & $2010 / 05 / 06$ & $J+N D_{\text {Short }}$ & $5.76 \pm 0.13$ \\
S13 & $2010 / 05 / 07$ & $J+N D_{\text {Short }}$ & $5.69 \pm 0.16$ \\
S13 & $2010 / 05 / 05$ & $H+N D_{\text {Short }}$ & $5.53 \pm 0.12$ \\
S13 & $2010 / 05 / 07$ & $H+N D_{\text {Short }}$ & $5.49 \pm 0.14$ \\
S27 & $2007 / 06 / 13$ & $K_{\mathrm{s}}+N D_{\text {Short }}$ & $5.37 \pm 0.15$ \\
S13 & $2009 / 09 / 28$ & $K_{\mathrm{s}}$ & $>5.0 \pm 0.1$ \\
S13 & $2010 / 05 / 05$ & $K_{\mathrm{s}}+N D_{\text {Short }}$ & $5.33 \pm 0.09$ \\
S13 & $2010 / 05 / 07$ & $K_{\mathrm{s}}+N D_{\text {Short }}$ & $5.35 \pm 0.11$ \\
\hline
\end{tabular}

Notes. The used filters, as well as the measured magnitude differences between PZTel B and its primary, are listed for all observing epochs.

individual observing epochs are all consistent with each other within their uncertainties.

The apparent magnitudes of PZTel B and its primary can be derived with the obtained magnitude-differences, as well as the accurate photometry of the PZTel system $(J=6.856 \pm$ $\left.0.021 \mathrm{mag}, H=6.486 \pm 0.049 \mathrm{mag}, K_{\mathrm{s}}=6.366 \pm 0.024 \mathrm{mag}\right)$, which is listed in the 2MASS point source catalogue (Skrutskie et al. 2006).

Finally, the precise Hipparcos parallax of PZ Tel yields a distance modulus $E=3.480 \pm 0.127 \mathrm{mag}$, which is used to derive the absolute magnitudes of PZ Tel B $\left(M_{J}=9.05 \pm 0.12 \mathrm{mag}\right.$, $M_{\mathrm{H}}=8.52 \pm 0.13 \mathrm{mag}$, and $\left.M_{K_{\mathrm{s}}}=8.24 \pm 0.10 \mathrm{mag}\right)$, assuming that the NACO and 2MASS $J H K_{\mathrm{s}}$ color systems are identical.

\section{Limits on additional companions}

We obtained deep NACO observations of PZ Tel A and its companion in September 2009 in the $K_{\mathrm{s}}$-band. The achieved detection limit of our NACO image with and without PSF subtraction is indicated in Fig. 3 together with the expected magnitudes of substellar objects with different masses at an assumed age of $10 \mathrm{Myr}$, derived with the Baraffe et al. (2003) evolutionary models and the distance of the PZ Tel system.

After PSF subtraction, all brown dwarf companions (mass $>$ $12 M_{\text {Jup }}$ ) are detectable in our NACO image beyond 0.23 arcsec (or $\sim 11 \mathrm{AU}$ of projected separation) around PZ Tel A up to about $7.7 \operatorname{arcsec}(\sim 380 \mathrm{AU})$ at the outer edge of the field of view, which was fully covered by NACO's S13 optics (jitter-width taken into account). In the background-noise-limited region, beyond a separation of about $1.75 \operatorname{arcsec}(\sim 87 \mathrm{AU})$, a sensitivity of $K_{\mathrm{s}}=19.6 \mathrm{mag}$ is reached in average, which allows the detection of planetary mass objects down to a mass of about $2 M_{\text {Jup }}$ around PZ Tel A.

Beside PZ Tel B, one additional very faint $\left(\Delta K_{\mathrm{s}}>10.2 \mathrm{mag}\right)$ companion-candidate is detected in our deep NACO image at sep $=3.818 \pm 0.006 \operatorname{arcsec}$ and $\mathrm{PA}=166.21 \pm 0.11^{\circ}$. This candidate was previously detected by Chauvin et al. (2010), who proposed that it is most likely a background source (Epoch: $2007 / 06 / 13$, sep $=4.008 \pm 0.008 \operatorname{arcsec}, \mathrm{PA}=166.3 \pm 0.2^{\circ}$, $\left.\Delta K_{\mathrm{s}}=10.7 \pm 0.1 \mathrm{mag}\right)$. If this object is a non-moving background object, we expect to find it at sep $=3.824 \pm 0.009$ arcsec, and PA $=165.80 \pm 0.21^{\circ}$ in September 2009. Our NACO astrometry of the candidate agrees well with the predicted values, hence this source is a slowly moving object, clearly not related to the PZTel system. Hence, on the basis of the achieved detection limit, we can conclude that there is no additional companion of PZ Tel A within a separation of $7.7 \operatorname{arcsec}(\sim 380 \mathrm{AU})$ around the star. However, we cannot exclude there being other 
A\&A 523, L1 (2010)

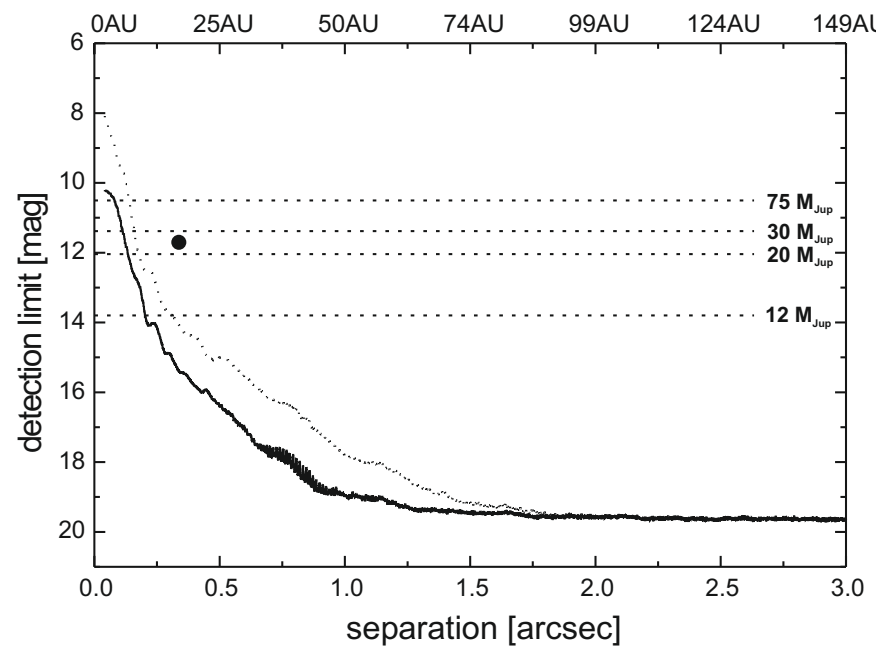

Fig. 3. The average detection limit of our deep NACO image taken in the $K_{\mathrm{s}}$-band in September 2009 plotted for a range of angular (bottom) and projected separation (top). The detection limit achieved in this image is shown as a dotted line, as well as the detection limit after PSF subtraction (solid line). Because of saturation, objects within a separation of 3 NACO pixels (or 40 mas of angular separation) cannot be detected around the bright primary. The position of PZTel B is indicated as black circle. Dashed horizontal lines show the expected brightness of substellar objects, whose masses are listed at the right side of the plot.

very close companions. Malaroda et al. (2000) list multi-epoch radial-velocity data of PZ Tel with a scatter of $4.4 \mathrm{~km} \mathrm{~s}^{-1}$, which could possibly be induced by another close companion or by the spots on the stellar surface (Barnes et al. 2000).

\section{Orbital motion}

Within all observing epochs from June 2007 to May 2010, both the separation and position angle of the PZ Tel B changed linearly with time at rates of sép $=34.7 \pm 1.0 \mathrm{mas} / \mathrm{yr}$ and $\dot{\mathrm{PA}}=$ $-0.41 \pm 0.08^{\circ} / \mathrm{yr}$. As PZ Tel B is located at an angular separation of $\sim 0.3$ arcsec on average, which corresponds to a projected separation of about $15 \mathrm{AU}$ at the distance of PZ Tel, the expected escape velocity is $\sim 46 \mathrm{mas} / \mathrm{yr}$, i.e. 1.3 times larger than the measured motion of the companion relative to its primary. Hence, the high relative motion of PZ Tel B (detected at a significance level $>37 \sigma$ ) is consistent with orbital motion of the companion around PZ Tel A. Between June 2007 and September 2009, the separation and the position angle of PZ Tel B change with slopes of sèp $=35.8 \pm 1.3 \mathrm{mas} / \mathrm{yr}$ and $\dot{\mathrm{PA}}=-0.5 \pm 0.3 \% \mathrm{yr}$ (see also Sect. 2), while we measure slopes of sep $=30.9 \pm 2.3 \mathrm{mas} / \mathrm{yr}$ and PA $=-0.2 \pm 0.4 \% \mathrm{yr}$ between September 2009 and May 2010 , i.e., smaller absolute values of both velocities in the second time interval. The relative motion of the companion decelerates slightly (at a $\sim 2 \sigma$ significance level), while its separation from its primary increases, in a way consistent with orbital motion. Because of the slow variation in PA relative to that of sep, the system is likely to be seen nearly edge-on.

With the obtained NACO detection limit, we conclude that PZTelB can be imaged with NACO (even without PSF subtraction) down to a separation of 0.16 arcsec around its much brighter primary. By tracing back the trajectory of the companion (derived from its relative motion), we expect PZ Tel B to have been detectable with NACO only since September 2004. Indeed, PZ Tel A was observed with NACO in July 2003, as reported by Masciadri et al. (2005), who also took deep $K_{\mathrm{s}}$-band images of the star, but could not detect its companion.

\section{Conclusions}

According to the evolutionary models of low-mass objects by Chabrier et al. (2000), Baraffe et al. (2002, 2003), at an age of $12_{-4}^{+8} \mathrm{Myr}$, the derived absolute infrared magnitudes of PZ Tel B are consistent with those of a brown dwarf with $28_{-4}^{+12} M_{\text {Jup }}$, and $T_{\text {eff }}=2500-2700 \mathrm{~K}$ (spectral type M6-8, Golimowski et al. 2004). Even if PZ Tel B were older than $20 \mathrm{Myr}$, it would still be within the substellar mass-regime, e.g., $69 M_{\text {Jup }}$ at $40 \mathrm{Myr}$. The model-predicted color $J-K_{\mathrm{s}}$ of such a brown dwarf is $J-K_{\mathrm{s}}=0.78 \pm 0.03 \mathrm{mag}$ (CIT-2MASS color transformation applied, Carpenter 2001), consistent with $J-K_{\mathrm{s}}=0.80 \pm 0.12 \mathrm{mag}$, as derived by us from our NACO photometry.

With only about $15 \mathrm{AU}$ of projected separation, PZ Tel B is the second closest substellar companion imaged directly around a young star after $\beta$ Pic b (Lagrange et al. 2010), i.e. much closer than e.g. the substellar companions of GQLup (Neuhäuser et al. 2005), CT Cha (Schmidt et al. 2008), or HR 7329 (Lowrance et al. 2000; Guenther et al. 2001). With $\beta$ Pic (Smith \& Terrile 1984) and HR 7329 (Smith et al. 2009), PZ Tel is the youngest star with both a substellar companion and a debris disk. All three stars are members of the $\beta$ Pic moving group, which contains a high fraction of stars with both debris disks and substellar companions. PZ Tel B is the first substellar companion imaged directly where orbital motion is detected (as deceleration) $)^{5}$.

Acknowledgements. We would like to thank the technical staff and all support astronomers at Paranal Observatory. We made use of the 2MASS public data releases, and the SIMBAD and VIZIER databases. NV acknowledges the support by projects DIPUV 07/2007 and Gemini-CONICYT 32090027. RN \& TOBS would like to thank DFG for support in program NE515/30-1.

\section{References}

Allende Prieto, C., \& Lambert, D. L. 1999, A\&A, 352, 555

Baraffe, I., Chabrier, G., Allard, F., \& Hauschildt, P. H. 2002, A\&A, 382, 563

Baraffe, I., Chabrier, G., Barman, T. S., Allard, F., \& Hauschildt, P. H. 2003, A\&A, 402, 701

Barnes, J. R., Collier Cameron, A., James, D. J., \& Donati, J. 2000, MNRAS, 314,162

Biller, B. A., Liu, M. C., Wahhaj, Z., et al. 2010, ApJ, 720, L82

Carpenter, J. M. 2001, AJ, 121, 2851

Chabrier, G., Baraffe, I., Allard, F., \& Hauschildt, P. 2000, ApJ, 542, 464

Chauvin, G., Lagrange, A., Bonavita, M., et al. 2010, A\&A, 509, A52

Devillard, N. 2001, in Astronomical Data Analysis Software and Systems X, ed F. R. Harnden Jr., F. A. Primini, \& H. E. Payne, SPC, 238, 525

Golimowski, D. A., Leggett, S. K., Marley, M. S., et al. 2004, AJ, 127, 3516

Guenther, E. W., Neuhäuser, R., Huélamo, N., Brandner, W., \& Alves, J. 2001, A\&A, 365, 514

Lagrange, A., Bonnefoy, M., Chauvin, G., et al. 2010, Science, 329, 57

Lowrance, P. J., Schneider, G., Kirkpatrick, J. D., et al. 2000, ApJ, 541, 390

Malaroda, S., Levato, H., Morrell, N., et al. 2000, A\&AS, 144, 1

Masciadri, E., Mundt, R., Henning, T., Alvarez, C., \& Barrado y Navascués, D. 2005, ApJ, 625, 1004

Neuhäuser, R., Guenther, E. W., Wuchterl, G., et al. 2005, A\&A, 435, L13

Perryman, M. A. C., Lindegren, L., Kovalevsky, J., et al. 1997, A\&A, 323, L49

Randich, S., Gratton, R., \& Pallavicini, R. 1993, A\&A, 273, 194

Rebull, L. M., Stapelfeldt, K. R., Werner, M. W., et al. 2008, ApJ, 681, 1484

Rocha-Pinto, H. J., Castilho, B. V., \& Maciel, W. J. 2002, A\&A, 384, 912

Schmidt, T. O. B., Neuhäuser, R., Seifahrt, A., et al. 2008, A\&A, 491, 31

Skrutskie, M. F., Cutri, R. M., Stiening, R., et al. 2006, AJ, 131, 1163

Smith, B. A., \& Terrile, R. J. 1984, Science, 226, 1421

Smith, R., Churcher, L. J., Wyatt, M. C., Moerchen, M. M., \& Telesco, C. M. 2009, A\&A, 493, 299

Soderblom, D. R., King, J. R., \& Henry, T. J. 1998, AJ, 116, 396

Tetzlaff, N., Neuhäuser, R., \& Hohle, M. M. 2010 [arXiv: 1007.4883]

Torres, C. A. O., Quast, G. R., da Silva, L., et al. 2006, A\&A, 460, 695

Zuckerman, B., Song, I., Bessell, M. S., \& Webb, R. A. 2001, ApJ, 562, L87

5 As this paper was about to be submitted, we became aware of a publication by Biller et al. (2010) whose results are completely consistent with those presented here. 